\title{
EXTREME TRANSPORT OF SEDIMENT DUE TO TURBIDITY CURRENTS IN COASTAL WATERS
}

\author{
LEO C. VAN RIJN \\ Delft Hydraulics, P.O.Box 177, 2600 MH Delft, The Netherlands,Leo.vanrijn@wldelft.nl \\ University of Utrecht, Phys. Geography, P.O. Box 80.115, 3508 TC Utrecht, Netherlands
}

\begin{abstract}
Turbidity currents of fine sand are analyzed and modelled using a depth-averaged approach for two layers (lower and upper layer). The basic equations and closure relationships for a turbidity current of fine sand are formulated and solved by means of a spreadsheet programme (Excel application). It is most easy to formulate the equations with respect to a tilting coordinate system assuming (1) that the flow is steady, (2) that the velocities $\left(\mathrm{u}_{1}\right)$ and sediment concentrations $\left(\mathrm{c}_{1}\right)$ in the upper layer 1 are negligibly small (density is equal to the fluid density), (3) that the flow in the lower layer 2 is fully turbulent and (4) that the pressure is hydrostatic. The present work was inspired by an extreme turbidity current event detected in the Zaire submarine canyon (Africa) at $4000 \mathrm{~m}$ water depth. This dataset was used to verify the numerical model.
\end{abstract}

\section{Introduction}

\subsection{Definitions}

Turbidity currents of fine sand and/or mud are sediment-laden underflows that can occur along relatively steep slopes and delta fronts in lakes, in reservoirs and in submarine canyons (Scripps canyon, California canyon in North America and Zaire canyon in Africa). Ignitive transformation of a delta foreset of fine sand along a submarine slope of fine sand may result into a density current in which the flow-energy substantially increases (self-acceleration). Often, these turbidity currents of fine sand are initiated by sudden failures (liquefaction, breaching or avalanching) of relatively steep bed slopes due to extreme shear forces.

High-density turbidity currents in marine conditions may also result from nonignitive conditions such as from high-concentration inflow of fine river sand and mud (hyperpycnal discharge) into the sea. Turbid river plumes (fine sand and mud) often enter the sea as intrusive plumes with an excess-density within the low-salinity values of coastal waters. These types of plumes will be rapidly diluted by mixing processes. In some cases river plumes can sustain by flowing along the seafloor (overcoming the shelf seawater densities). This latter phenomenon is known as a hyperpycnal flow (negatively bouyant plume that flows along the seabed due to plume sediment density in excess of the ambient density). The critical concentration in a river needed to produce a hyperpycnal plume in marine coastal water is relatively large $\left(25\right.$ to $\left.50 \mathrm{~kg} / \mathrm{m}^{3}\right)$. All over the earth there are about 10 high-turbid rivers with average concentrations larger 
than $10 \mathrm{~kg} / \mathrm{m}^{3}$. Except for the Huanghe (Yellow) River in China, most of these rivers drain small mountainous areas with low annual discharge.

Field observations show that turbidity currents can attain relatively high velocities up to $10 \mathrm{~m} / \mathrm{s}$. High velocities are presumably attained as the result of acceleration from some lower velocity caused by an initial disturbance. The energy for acceleration is derived from the sediment itself (work performed by the downstream component of gravity on the suspended sediments). Hence, the turbid sediment layer drags the water along (self acceleration). If the velocity is sufficiently high, it will erode more sediment from the bed into the turbidity layer, thus increasing the rate of work performed by gravity resulting into increased acceleration (self-reinforcing process). According to Bagnold (1962), a self-sustaining turbidity current can be generated if: $u \sin \beta / w_{s}>1$ (with $u=$ velocity, $\beta=$ slope angle and $\mathrm{w}_{\mathrm{s}}=$ settling velocity of sediment). The velocity and sediment concentration might rise indefinitely because more energy is fed into the turbidity layer than is expended in holding the sediments in suspension. The effect of damping of turbulence by the sediment particles is, however, not taken into account by this criterion $\left(u \sin \beta / w_{s}>1\right)$. If the turbulence is reduced due to the presence of sediments, part of the sediment particles will settle out and hence the energy for driving the turbidity current will be reduced resulting in a decrease of the velocity of the turbidity current.

\subsection{Observations in Zaire canyon, Africa}

The present work was inspired by an extreme turbidity current event detected in the Zaire submarine canyon at $4000 \mathrm{~m}$ water depth (Khripounoff, 2003). Current meters, turbidity meters and sediment traps deployed on a mooring cable located in the canyon axis, although they were damaged, recorded the signature of a very extreme event. The Zaire canyon (depth of about $150 \mathrm{~m}$ below the surrounding bottom, width of 1 to $2 \mathrm{~km}$ ) extends over about $760 \mathrm{~km}$ from the river mouth to the ocean floor (water depth of $5100 \mathrm{~m}$ ) downslope from the Congo-Angola continental shelf. In January 2001, two instrumented moorings were deployed at $4000 \mathrm{~m}$ water depth. Data from the two current meters and the turbiditymeter moored in the canyon showed no particular events (almost zero-velocities) during the first three months. At $22.00 \mathrm{hrs}$ on March 8 (2001) a sudden pulse was recorded by both current meters and a strong increase of turbidity was measured. On the next record (1 hour later) the lower current meter was damaged but the upper current meter recorded a value of 1.2 $\mathrm{m} / \mathrm{s}$ at about 110 to $150 \mathrm{~m}$ above the bottom (average over 1 hour; undervalued due to tilting cable). The sample bottles were filled with fine sand ( 0.15 to 0.2 $\mathrm{mm}$ ) and debris. More data were not recorded as the mooring cable was broken around 24 hours of March 8 . At station 2 outside the canyon, the turbidity current was observed about three days later (by overflow from the canyon); a bottle at $30 \mathrm{~m}$ above the bed was filled with brown clay. The turbidity meter at $400 \mathrm{~m}$ above the bed did not show any signs of increased turbidity levels 
indicating that the turbidity current overflow remained in the near-bed region $(<$ $400 \mathrm{~m})$.

\section{Basic Equations}

\subsection{Two-layer schematization}

The basic equations for a non-steady turbidity current in three-dimensional and two-dimensional depth-averaged conditions are given by Van Rijn (2005). Similar equations have been formulated by Parker et al. (1986) and by Sloff (1994, 1997).

In the case of a $1 \mathrm{D}$ channel with a constant slope angle $(\beta)$ it is most easy to formulate the equations with respect to a tilting coordinate system (,$n$ plane with slope angle $\beta$, see Figure 1). The hydrostatic pressure is now defined along the $n$-axis, yielding a gcos $\beta$-term (for all terms with $\partial \mathrm{h}_{2} / \partial \mathrm{s}$ ) and a gsin $\beta$-term for the slope force along the s-axis (decomposition of gravity force vector). Assuming (1) that the flow is steady, (2) that the velocities $\left(\mathrm{u}_{1}\right)$ and sediment concentrations $\left(\mathrm{c}_{1}\right)$ in the upper layer are negligibly small (density is equal to the fluid density), (3) that the flow in the lower layer is fully turbulent and (4) that the pressure is hydrostatic, the equations for $1 \mathrm{D}$ conditions can be expressed by Eqs. (1) to (.3).

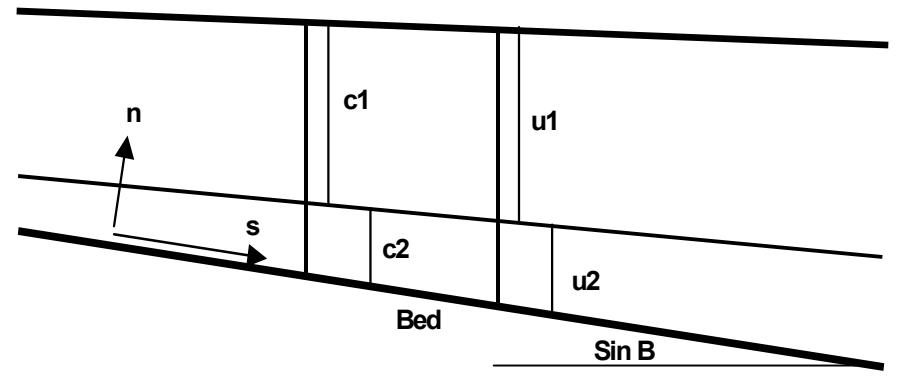

Figure 1 Tilting coordinate system for schematized turbidity current (1D) in vertical plane

momentum balance of mixture in lower layer 2 in s-direction

$\rho_{2} \partial\left(\mathrm{u}_{2}{ }^{2} \mathrm{~h}_{2}\right) / \partial \mathrm{s}+\left(\rho_{\mathrm{s}}-\rho_{\mathrm{w}}\right) \mathrm{h}_{2} \mathrm{c}_{2}\left[(\mathrm{~g} \cos \beta) \partial \mathrm{h}_{2} / \partial \mathrm{s}\right.$

$-g \sin \beta]+\left(\rho_{\mathrm{s}}-\rho_{\mathrm{w}}\right)\left(0.5 \mathrm{gh}_{2}{ }^{2} \cos \beta+\mathrm{u}_{2}{ }^{2} \mathrm{~h}_{2}\right) \partial \mathrm{c}_{2} / \partial \mathrm{s}+\left(\tau_{\mathrm{i}}+\tau_{\mathrm{b}}\right)=0$ 
mass balance for fluid in lower layer 2

$\partial\left(\mathrm{u}_{2} \mathrm{~h}_{2}\left(1-\mathrm{c}_{2}\right)\right) / \partial \mathrm{s}-\mathrm{W}_{\mathrm{i}}-\mathrm{W}_{\mathrm{b}}=0$

mass balance for sediment in lower layer 2

$\partial\left(\mathrm{u}_{2} \mathrm{c}_{2} \mathrm{~h}_{2}\right) / \partial \mathrm{s}-\mathrm{S}_{\mathrm{i}}-\mathrm{S}_{\mathrm{b}}=0$

with :

$\mathrm{h}_{1}, \mathrm{~h}_{2}=$ thickness of upper and lower layer $\left(\mathrm{h}_{1}+\mathrm{h}_{2}=\mathrm{h}=\right.$ flow depth $)$,

$\mathrm{c}_{1}, \mathrm{c}_{2}=$ depth-averaged volumetric suspended sediment concentration in upper layer 1 and lower layer 2 ,

$\mathrm{u}_{1}=\mathrm{q}_{1} / \mathrm{h}_{1}, \mathrm{u}_{2}=\mathrm{q}_{2} / \mathrm{h}_{2}=$ velocity in upper layer 1 and lower layer 2 ,

$\mathrm{W}_{\mathrm{i}}=$ exchange of fluid at the interface,

$\mathrm{W}_{\mathrm{b}}=$ exchange of fluid at the bed,

$\mathrm{S}_{\mathrm{i}}=$ exchange of sediment at the interface,

$\mathrm{S}_{\mathrm{b}}=$ exchange of sediment at the bed,

$\rho_{2}=$ mixture density of lower layer,

$\rho_{\mathrm{w}}=$ fluid density (clear water in upper layer 1),

$\rho_{\mathrm{s}}=$ sediment density,

$\tau_{\mathrm{i}}=$ shear stress at interface $\left(=\rho \mathrm{C}_{\mathrm{di}} \mathrm{u}_{2}{ }^{2}\right)$,

$\tau_{\mathrm{b}}=$ bed shear stress $\left(=\rho \mathrm{C}_{\mathrm{d}} \mathrm{u}_{2}^{2}\right)$,

$\mathrm{C}_{\mathrm{d}}=$ bottom friction coefficient $\left(=\mathrm{g} / \mathrm{C}^{2}\right), \mathrm{C}=$ Chézy coefficient,

$\mathrm{C}_{\mathrm{di}}=$ interface friction coeffcient,

$\beta=$ angle of bed slope in s-direction,

$\mathrm{s}=$ coordinate along bed slope.

The equations (1), (2) and (3) define a set of three equations with three unknown parameters $\mathrm{u}_{2}, \mathrm{~h}_{2}$ and $\mathrm{c}_{2}$, which can be solved for given boundary conditions. The closure expressions are given below. Similar equations have been formulated by Parker et al. (1986), Akiyama and Stefan (1985) and by Mastbergen and Van den Berg (2003).

Equations (1) and (2) can be reformulated (eliminating the term $\partial \mathrm{u}_{2} / \partial \mathrm{s}$ using Eq. 2), yielding:

$\partial \mathrm{h}_{2} / \partial \mathrm{s}=\left[1 /\left(\gamma_{2}\left(1-\mathrm{c}_{2}\right)\right)\right]\left[\gamma_{1}\left(1-\mathrm{c}_{2}\right)-\left(1-\mathrm{c}_{2}\right)\left(\tau_{\mathrm{i}}+\tau_{\mathrm{b}}\right)\right.$

$\left.-2 \rho_{2} \mathrm{u}_{2}\left(\mathrm{~W}_{\mathrm{i}}+\mathrm{W}_{\mathrm{b}}\right)-\gamma_{3} \partial \mathrm{c}_{2} / \partial \mathrm{s}\right]$

with:

$\gamma_{1}=\left(\rho_{\mathrm{s}}-\rho_{\mathrm{w}}\right) \mathrm{h}_{2} \mathrm{c}_{2} \mathrm{~g} \sin \beta$

$\gamma_{2}=\left(\rho_{\mathrm{s}}-\rho_{\mathrm{w}}\right) \mathrm{h}_{2} \mathrm{c}_{2} \mathrm{~g} \cos \beta-\rho_{2}\left(\mathrm{u}_{2}\right)^{2}=\left(\rho_{\mathrm{s}}-\rho_{\mathrm{w}}\right) \mathrm{h}_{2} \mathrm{c}_{2} \mathrm{~g} \cos \beta\left[1-\left(\mathrm{h}_{2, \mathrm{cr}} / \mathrm{h}_{2}\right)^{3}\right]$

$\gamma_{3}=2 \rho_{2} h_{2}\left(u_{2}\right)^{2}+\left(\rho_{s}-\rho_{\mathrm{w}}\right)\left(1-c_{2}\right) h_{2}\left(u_{2}\right)^{2}+0.5\left(\rho_{s}-\rho_{\mathrm{w}}\right)\left(1-c_{2}\right)\left(h_{2}\right)^{2} g \cos \beta$

Equation (4) can be used for all slope values (including $\beta=0$ ). 
Defining a critical depth $\left(\mathrm{Fr}_{2}=1\right)$ and an equilibrium depth (uniform flow of water and sediment; $\left.\tau_{\mathrm{i}}+\tau_{\mathrm{b}}=\rho_{2}\left(\mathrm{C}_{\mathrm{d}}+\mathrm{C}_{\mathrm{di}}\right) \mathrm{q}^{2} / \mathrm{h}_{2}{ }^{2}\right)$, Equation (4) can also be expressed as:

$\partial \mathrm{h}_{2} / \partial \mathrm{s}=\frac{(\sin \beta / \cos \beta)\left[1-\left(\mathrm{h}_{2, \mathrm{eq}} / \mathrm{h}_{2}\right)^{3}-\alpha_{1}\left(\mathrm{~W}_{\mathrm{i}}+\mathrm{W}_{\mathrm{b}}\right) / \mathrm{u}_{2}-\alpha_{2} \mathrm{~h}_{2} \partial \mathrm{c}_{2} / \partial \mathrm{s}\right]}{\left[1-\left(\mathrm{h}_{2, \mathrm{cr}} / \mathrm{h}_{2}\right)^{3}\right]}$

with:

$\alpha_{1}=\frac{2 \rho_{2}\left(u_{2}\right)^{2}}{\left(\rho_{s}-\rho_{w}\right)\left(1-c_{2}\right) h_{2} c_{2} g \sin \beta}$
$\alpha_{2}=\frac{2 \rho_{2}\left(u_{2}\right)^{2}+\left(\rho_{s}-\rho_{w}\right)\left(1-c_{2}\right)\left(u_{2}\right)^{2}+0.5\left(\rho_{s}-\rho_{w}\right)\left(1-c_{2}\right) h_{2} g \cos \beta}{\left(\rho_{s}-\rho_{w}\right)\left(1-c_{2}\right) c_{2} h_{2} g \sin \beta}$

$\mathrm{h}_{2, \mathrm{cr}}=\left[\mathrm{q}^{2} /\left\{\left(\left(\rho_{\mathrm{s}}-\rho_{\mathrm{w}}\right) / \rho_{2}\right) \mathrm{c}_{2} \mathrm{~g} \cos \beta\right\}\right]^{1 / 3}=$ critical depth (defined by Froude number $\left.\mathrm{Fr}_{2}=1\right)$

$\mathrm{h}_{2, \mathrm{eq}}=\left[\left(\mathrm{C}_{\mathrm{d}}+\mathrm{C}_{\mathrm{di}}\right) \mathrm{q}^{2} /\left\{\left(\left(\rho_{\mathrm{s}}-\rho_{\mathrm{w}}\right) / \rho_{2}\right) \mathrm{c}_{2} \mathrm{~g} \sin \beta\right\}\right]^{1 / 3}=$ equilibrium depth (uniform turbulent flow of water and sediment)

$\mathrm{h}_{2, \mathrm{eq}}=\left[\left(\tau_{\mathrm{I}}+\tau_{\mathrm{b}}\right)\left(\mathrm{h}_{2}\right)^{2} /\left(\left(\rho_{\mathrm{s}}-\rho_{\mathrm{w}}\right) \mathrm{c}_{2} \mathrm{~g} \sin \beta\right)\right]^{1 / 3} \quad=$ equilibrium depth (uniform laminar flow of water and sediment)

$\tau_{\mathrm{b}}+\tau_{\mathrm{i}}=\rho_{2}\left(\mathrm{C}_{\mathrm{d}}+\mathrm{C}_{\mathrm{di}}\right) \mathrm{q}^{2} / \mathrm{h}_{2}^{2}=$ shear stress for turbulent flow $\left(\mathrm{C}_{\mathrm{di}}=0.333 \mathrm{C}_{\mathrm{d}}\right)$

$\tau_{\mathrm{b}}=3 v_{2} \rho_{2} \mathrm{q} / \mathrm{h}_{2}^{2} \quad=$ bed-shear stress for laminar flow $\left(\tau_{\mathrm{i}}=0.333 \tau_{\mathrm{b}}\right)$

Equation (5) can not be used for a slope of $\beta=0$, because $h_{2 \text {,eq }}$ is undefined for slope $\beta=0$. Assuming supercritical flow $\left(\mathrm{Fr}_{2}>1\right)$, the gradient $\partial \mathrm{h}_{2} / \partial$ s increases $\left(\mathrm{h}_{2}\right.$ grows more rapidly) with:

- increasing entrainment $\left(\mathrm{W}_{\mathrm{i}}+\mathrm{W}_{\mathrm{b}}\right)$ of fluid into the lower layer;

- increasing shear stresses $\left(\tau_{\mathrm{i}}+\tau_{\mathrm{b}}\right)$ at the bed and at the interface;

- increasing concentration in downstream direction (increasing $\partial \mathrm{c}_{2} / \partial \mathrm{s}$ ).

A special case is obtained by neglecting the exchange terms at the upper interface and at the bed $\left(\mathrm{W}_{\mathrm{i}}=\mathrm{W}_{\mathrm{b}}=\mathrm{S}_{\mathrm{i}}=\mathrm{S}_{\mathrm{b}}=0\right)$. Consequently, it follows that $\partial \mathrm{q} / \partial \mathrm{s}=0$ and $\partial \mathrm{q}_{\mathrm{s}} / \partial \mathrm{s}=0$ and thus $\partial \mathrm{c}_{2} / \partial \mathrm{s}=0$, yielding (see also Ning Chien, 1999):

$\partial \mathrm{h}_{2} / \partial \mathrm{s}=\frac{(\sin \beta / \cos \beta)\left[1-\left(\mathrm{h}_{2, \mathrm{eq}} / \mathrm{h}_{2}\right)^{3}\right]}{\left[1-\left(\mathrm{h}_{2, \mathrm{cr}} / \mathrm{h}_{2}\right)^{3}\right]}$ 
Equation (6) shows a close resemblance with the Bélanger equation for clear water flow (Van Rijn, 1990).

For supercritical flow $\left(\mathrm{Fr}_{2}>1\right)$ with $\mathrm{h}_{2}<\mathrm{h}_{2 \text {,eq }}$ and $\mathrm{h}_{2}<\mathrm{h}_{2 \text {,cr }}$ it follows that the upper and lower terms of Equations (5) and (6) are both negative and thus $\partial \mathrm{h}_{2} / \partial \mathrm{s}>0$ or a growing layer thickness in downstream direction.

Equations (5) and (6) are only meaningful for a supercritical turbidity current. In subcritical conditions the sediment particles will rapidly settle out resulting in a 'normal' Rouse-type of concentration profile.

The mass balances of fluid and sediment (Eqs. 2 and 3) can be reformulated as:

$\partial \mathrm{q} / \partial \mathrm{s}=\partial \mathrm{q}_{\mathrm{s}} / \partial \mathrm{s}+\left(\mathrm{W}_{\mathrm{i}}+\mathrm{W}_{\mathrm{b}}\right)$

$\partial \mathrm{q}_{\mathrm{s}} / \partial \mathrm{s}=\mathrm{S}_{\mathrm{b}}+\mathrm{S}_{\mathrm{i}}$

with:

$\mathrm{u}_{2}=\mathrm{q} / \mathrm{h}_{2}$

$\mathrm{c}_{2}=\mathrm{q}_{\mathrm{s}} / \mathrm{q}$

\subsection{Densimetric parameters}

The density difference between the sediment suspension in the lower layer 2 and the clear water is given by:

$\Delta \rho=\rho_{2}-\rho_{\mathrm{w}}=\left[\rho_{\mathrm{s}} \mathrm{c}_{2}+\left(1-\mathrm{c}_{2}\right) \rho_{\mathrm{w}}\right]-\rho_{\mathrm{w}}=\mathrm{c}_{2}\left(\rho_{\mathrm{s}}-\rho_{\mathrm{w}}\right)$

The relative density difference is given by:

$\Delta \rho / \rho_{\mathrm{w}}=\mathrm{c}_{2}\left(\rho_{\mathrm{s}}-\rho_{\mathrm{w}}\right) / \rho_{\mathrm{w}}=\mathrm{c}_{2}(\mathrm{~s}-1)=\left(\mathrm{c}_{2 \mathrm{~m}} / \rho_{\mathrm{s}}\right)(\mathrm{s}-1)$

or by:

$\Delta \rho / \rho_{2}=\left[c_{2}\left(\rho_{\mathrm{s}}-\rho_{\mathrm{w}}\right)\right] /\left[\mathrm{c}_{2}\left(\rho_{\mathrm{s}}-\rho_{\mathrm{w}}\right)+\rho_{\mathrm{w}}\right]=\mathrm{c}_{2}(\mathrm{~s}-1) /\left(\mathrm{c}_{2}(\mathrm{~s}-1)+1\right)$

with:

$\rho_{2}=\rho_{\mathrm{s}} c_{2}+\left(1-c_{2}\right) \rho_{\mathrm{w}}=$ density of fluid-sediment mixure of lower turbidity layer,

$c_{2}=\left(\rho_{2}-\rho_{\mathrm{w}}\right) /\left(\rho_{\mathrm{s}}-\rho_{\mathrm{w}}\right)=$ volume concentration of lower layer,

$c_{2 m}=c_{2} \rho_{s}=\rho_{s}\left(\rho_{2}-\rho_{w}\right) /\left(\rho_{s}-\rho_{w}\right)=$ mass concentration of lower layer,

$\rho_{\mathrm{w}}=$ fluid density (fresh water $=1000 \mathrm{~kg} / \mathrm{m}^{3}$, saline water $=1020 \mathrm{~kg} / \mathrm{m}^{3}$ ),

$\rho_{\mathrm{s}}=$ sediment density $\left(2650 \mathrm{~kg} / \mathrm{m}^{3}\right)$,

$\mathrm{s} \quad=$ relative density $=\rho_{\mathrm{s}} / \rho_{\mathrm{w}}$. 
Equation (13) reduces to Equation (12) for low concentrations $(<0.01$ or approx. $\left.25 \mathrm{~kg} / \mathrm{m}^{3}\right)$.

\subsection{Closure expressions for turbidity currents of fine sand}

The water exchange processes are represented as:

$\mathrm{W}_{\mathrm{i}}=\mathrm{w}_{\mathrm{ie}}\left(1-\mathrm{c}_{1}\right)=$ net water exchange rate at upper interface $\mathrm{z}_{\mathrm{i}}\left(\mathrm{w}_{\mathrm{ie}}\right.$ is directed downwards),

$\mathrm{w}_{\mathrm{ie}}=$ vertical exchange water velocity at upper interface $\mathrm{z}_{\mathrm{i}}$ (positive downward velocity $)=\left(0.002 / \mathrm{Ri}_{\mathrm{o} 2}\right) \mathrm{u}_{2}$ for $0.1<\mathrm{Ri}_{\mathrm{o} 2}<10$,

$\mathrm{Ri}_{\mathrm{o} 2}=\left[\mathrm{g}\left(\rho_{2}-\rho_{\mathrm{w}}\right) / \mathrm{h}_{2}\right] /\left[\rho_{2}\left(\mathrm{u}_{2} / \mathrm{h}_{2}\right)^{2}\right]=$ bulk-Richardson number of lower layer 2 ,

$\mathrm{W}_{\mathrm{b}}=\eta \mathrm{v}_{\mathrm{b}, \mathrm{e}}=$ vertical water supply from bed into lower layer (positive upwards;

$\mathrm{W}_{\mathrm{b}}=0$, if $\mathrm{S}_{\mathrm{b}}=\mathrm{O}$ )),

$\mathrm{v}_{\mathrm{b}, \mathrm{e}}=$ erosion velocity at bed surface (positive upwards).

The sediment exchange processes are represented as:

$\mathrm{S}_{\mathrm{i}}=$ net sediment exchange rate at interface $=-\mathrm{V}_{\mathrm{i}, \text { up }}+\mathrm{V}_{\mathrm{i} \text {,down }}=$

$-\varepsilon_{\mathrm{si}}\left(\mathrm{c}_{\mathrm{i}} / \mathrm{h}_{2}\right)+\left(\mathrm{w}_{\mathrm{ie}}+\mathrm{w}_{\mathrm{s}, \mathrm{o}}\right) \mathrm{c}_{1}$ for sandy bed,

$\mathrm{V}_{\mathrm{i}, \text { down }}=$ downward sediment exchange velocity $=\left(\mathrm{w}_{\mathrm{ie}}+\mathrm{w}_{\mathrm{s}, \mathrm{o}}\right) \mathrm{c}_{1}$,

$\mathrm{V}_{\mathrm{i}, \mathrm{up}}=$ upward sediment exchange velocity $=\varepsilon_{\mathrm{si}}\left(\mathrm{c}_{\mathrm{i}} / \mathrm{h}_{2}\right)=$

$0.05 \alpha_{\mathrm{d}} \mathrm{u}_{*} \mathrm{c}_{\mathrm{i}}=0.025 \alpha_{\mathrm{d}} \mathrm{u}_{*} \mathrm{c}_{2}$,

$\varepsilon_{\mathrm{si}}=$ mixing coefficient at interface $=0.05 \mathrm{u} * \mathrm{~h}_{2}$,

$\mathrm{c}_{\mathrm{i}}=$ concentration at interface (assumed to be $0.5 \mathrm{c}_{2}$ ),

$\alpha_{\mathrm{d}}=$ coefficient related to damping of turbulence ( 0 to 1 , input value),

$\mathrm{S}_{\mathrm{b}}=\mathrm{V}_{\mathrm{b}, \mathrm{e}}-\mathrm{V}_{\mathrm{b} \text {,sed }}=$ net sediment exchange velocity at bed, $\left(\mathrm{S}_{\mathrm{b}}=0\right.$ for flow over a rocky bed), $\left(\mathrm{S}_{\mathrm{b}}=0\right.$ for $\left.\mathrm{c}_{2}>\mathrm{c}_{2, \max }\right)$

$\mathrm{V}_{\mathrm{b}, \mathrm{e}}=$ erosion velocity at bed $=(1-\eta) \mathrm{v}_{\mathrm{b}, \mathrm{e}}$ for sandy bed (positive upwards), (incl. porosity to obtain pure sediment volume in lower layer),

$\mathrm{V}_{\mathrm{b}, \mathrm{sed}}=\mathrm{w}_{\mathrm{s}} \mathrm{c}_{\mathrm{b}}=\mathrm{w}_{\mathrm{s}, \mathrm{o}}\left(1-\mathrm{c}_{\mathrm{b}}\right)^{4} \mathrm{c}_{\mathrm{b}}=$ downward deposition velocity at bed (deposition), $\mathrm{c}_{2, \max }=$ maximum concentration in lower layer (input value),

$\mathrm{c}_{\mathrm{b}}=$ near-bed concentration (assumed to be $2 \mathrm{c}_{2}$ ),

$\mathrm{W}_{\mathrm{s}, \mathrm{o}}=$ sediment fall velocity of a single particle in clear water (positive downwards),

$\mathrm{w}_{\mathrm{s}}=\mathrm{w}_{\mathrm{s}, \mathrm{o}}\left(1-\mathrm{c}_{\mathrm{b}}\right)^{4}=$ sediment fall velocity of sediment in a mixture,

The erosion velocity at the bed can be described by:

$\mathrm{v}_{\mathrm{b}, \mathrm{e}}=\alpha_{\mathrm{e}}\left[(\mathrm{s}-1) \mathrm{gd}_{50}\right]^{0.5}\left[\theta-\theta_{\mathrm{cr}}\right]^{0.5} \mathrm{D}^{0.3} / \Psi$

$\mathrm{v}_{\mathrm{b}, \mathrm{e}}=0$ for $\theta<\theta_{\mathrm{cr}}$

$\left.\mathrm{v}_{\mathrm{b}, \mathrm{e}, \max }=0.033\left[\left(\mathrm{c}_{2 \max } / \mathrm{c}_{2}\right)-1\right)\right]\left[(\mathrm{s}-1) \mathrm{gd}_{50}\right]^{0.5}$ (Winterwerp et al., 1992)

with: $s=\rho_{\mathrm{s}} / \rho_{\mathrm{w}}=$ relative density, $\eta=$ bed porosity factor $(=0.4), \mathrm{D}_{*}=\mathrm{d}_{50}((\mathrm{~s}-$ $\left.1) \mathrm{g} / \mathrm{v}^{2}\right)^{1 / 3}=$ dimensionless particle size parameter, $\theta=\tau_{\mathrm{b}} /\left(\left(\rho_{\mathrm{s}}-\rho_{\mathrm{w}}\right) g \mathrm{gd}_{50}\right)=$ particle 
mobility number, $\tau_{b}=$ bed shear stress, $\Psi=$ slope factor $=1-(\sin \beta / \sin \phi), \beta=$ bed slope angle, $\phi=$ static bed friction angle (angle of repose), $\theta_{\mathrm{cr}}=$ critical mobility parameter according to Shields, $\alpha_{\mathrm{e}}=$ coefficient in the range of 0.002 to 0.003 , $\mathrm{c}_{2 \max }=$ maximum concentration in lower layer $(=0.6)$.

Computed (based on $\alpha_{\mathrm{e}}=0.0025$ ) and measured erosion velocities (Van Rijn, 1983, 1984, 1986; Winterwerp et al., 1992) in the case of a bed of fine sand are given in Table 1 . The agreement is reasonably good for low velocities smaller than $0.7 \mathrm{~m} / \mathrm{s}$. The computed erosion velocities are too small (factor 3 ) for larger velocities.

\begin{tabular}{|c|c|c|c|c|}
\hline \multirow{2}{*}{$\begin{array}{l}\text { Velocity } u_{2} \\
(\mathrm{~m} / \mathrm{s})\end{array}$} & \multicolumn{2}{|r|}{ Erosion } & \multicolumn{2}{|c|}{ velocity $v_{b e}(\mathrm{~mm} / \mathrm{s})$} \\
\hline & $\begin{array}{l}\text { Computed } \\
(0.11 \mathrm{~mm})\end{array}$ & $\begin{array}{c}\text { Measured } \\
(0.12 \text { to } 0.13 \mathrm{~mm})\end{array}$ & $\begin{array}{l}\text { Computed } \\
(0.25 \mathrm{~mm})\end{array}$ & $\begin{array}{r}\text { Measured } \\
(0.19 \text { to } 0.36 \mathrm{~mm})\end{array}$ \\
\hline 0.3 & 0.084 & & 0.094 & \\
\hline 0.5 & 0.15 & $0.1(0.13 \mathrm{~mm})$ & 0.18 & $0.1(0.19 \mathrm{~mm})$ \\
\hline 0.7 & 0.21 & $0.4(0.13 \mathrm{~mm})$ & 0.25 & $0.3(0.19 \mathrm{~mm})$ \\
\hline 1.0 & 0.30 & 1 to $2(0.12 \mathrm{~mm})$ & 0.37 & $\begin{array}{r}0.8 \text { to } 1.7(0.19 \mathrm{~mm}) \\
1(0.36 \mathrm{~mm})\end{array}$ \\
\hline 1.5 & 0.46 & $2(0.12 \mathrm{~mm})$ & 0.56 & \\
\hline 2.0 & 0.61 & 3 to $5(0.12 \mathrm{~mm})$ & 0.75 & \\
\hline 2.5 & 0.75 & & 0.93 & 3 to $4(0.225 \mathrm{~mm})$ \\
\hline 3.0 & 0.92 & & 1.10 & \\
\hline
\end{tabular}

Table 1 Computed and measured erosion velocities for two grain diameters $\left(d_{50}=0.11\right.$ and $\left.0.25 \mathrm{~mm}\right)$

\subsection{Auto-suspending turbidity currents}

Auto-suspending turbidity currents are defined as turbidity currents in which the rate of sediment entrainment equals or exceeds the rate of sediment deposition so that a steady or accelerating flow is maintained and that the density difference and the bed slope are such that the downslope force equals or exceeds the resistance forces.

Assuming equilibrium conditions in the lower layer, the velocity $\mathrm{u}_{2}$ can be estimated from Equation (1), see also $\mathrm{h}_{2, \mathrm{eq}}$ of Equation (5), yielding:

$\mathrm{u}_{2}=\left[\left(\left(\left(\rho_{\mathrm{s}}-\rho_{\mathrm{w}}\right) / \rho_{2}\right) \mathrm{c}_{2} \mathrm{gh}_{2} \sin \beta\right) /\left(\mathrm{C}_{\mathrm{d}}+\mathrm{C}_{\mathrm{di}}\right)\right]^{0.5}=\left[\left(\left(\Delta \rho / \rho_{2}\right) \operatorname{gh}_{2} \sin \beta\right) /\left(\mathrm{C}_{\mathrm{d}}+\mathrm{C}_{\mathrm{di}}\right)\right]^{0.5}$

with: $\sin \beta=$ bottom slope, $h_{2}=$ thickness of lower layer, $c_{2}=$ volumetric depthaveraged concentration in lower layer, $\mathrm{s}=$ relative density $=1.65, \Delta \rho=\left(\rho_{\mathrm{s}}-\rho_{\mathrm{w}}\right) \mathrm{c}_{2}$, $\rho_{2}=\rho_{\mathrm{s}} \mathrm{c}_{2}+\left(1-\mathrm{c}_{2}\right) \rho_{\mathrm{w}}, \quad \Delta \rho / \rho_{2}=\mathrm{c}_{2}(\mathrm{~s}-1) /\left(\mathrm{c}_{2}(\mathrm{~s}-1)+1\right), \quad \mathrm{C}_{\mathrm{d}}=$ bed friction coefficient $\left(=\mathrm{g} / \mathrm{C}^{2} \cong 0.0023\right.$ for Chézy $\left.\mathrm{C}=65 \mathrm{~m}^{0.5} / \mathrm{s}\right), \mathrm{C}_{\mathrm{di}}=$ interface-friction coefficient $(\cong 1 / 3$ $\mathrm{C}_{\mathrm{d}}$ ). Some values are given below: 
Using $\mathrm{h}_{2}=1 \mathrm{~m}, \mathrm{c}_{2}=0.001\left(=2.65 \mathrm{~kg} / \mathrm{m}^{3}\right), \sin \beta=0.001$ ( 1 to 1000$), \mathrm{C}_{\mathrm{d}}+\mathrm{C}_{\mathrm{di}}=0.003$, it follows that: $\mathrm{u}_{2} \cong 0.073 \mathrm{~m} / \mathrm{s}$.

Using $\mathrm{h}_{2}=1 \mathrm{~m}, \mathrm{c}_{2}=0.01 \quad\left(=26.5 \mathrm{~kg} / \mathrm{m}^{3}\right), \sin \beta=0.001$ ( 1 to 1000$), \mathrm{C}_{\mathrm{d}}+\mathrm{C}_{\mathrm{di}}=0.003$, it follows that: $\mathrm{u}_{2} \simeq 0.23 \mathrm{~m} / \mathrm{s}$.

Using $\mathrm{h}_{2}=1 \mathrm{~m}, \mathrm{c}_{2}=0.1 \quad\left(=265 \mathrm{~kg} / \mathrm{m}^{3}\right), \quad \sin \beta=0.001$ ( 1 to 1000$), \mathrm{C}_{\mathrm{d}}+\mathrm{C}_{\mathrm{di}}=0.003$, it follows that: $\mathrm{u}_{2} \cong 0.68 \mathrm{~m} / \mathrm{s}$.

Using $\mathrm{h}_{2}=1 \mathrm{~m}, \mathrm{c}_{2}=0.01 \quad\left(=26.5 \mathrm{~kg} / \mathrm{m}^{3}\right), \sin \beta=0.01 \quad(1$ to 100$), \mathrm{C}_{\mathrm{d}}+\mathrm{C}_{\mathrm{di}}=0.003$, it follows that: $\mathrm{u}_{2} \cong 0.73 \mathrm{~m} / \mathrm{s}$.

Equation (15) yields a value of about $0.3 \mathrm{~m} / \mathrm{s}$ for the turbidity current in the Sanmenxia reservoir in China, using $\mathrm{C}_{\mathrm{d}}=0.001$ (plane bed without bed forms, $\mathrm{C}=100 \mathrm{~m} / \mathrm{s} / \mathrm{s}), \mathrm{h}_{2}=3 \mathrm{~m}, \sin \beta=1 / 5000, \mathrm{c}_{2}=20 \mathrm{~kg} / \mathrm{m}^{3}$ or $\rho_{2}=1012 \mathrm{~kg} / \mathrm{m}^{3}$ $\left(\Delta \rho / \rho_{\mathrm{w}}=0.0124\right.$ and $\left.\Delta \rho / \rho_{2}=0.0118\right)$. The measured velocities of the turbidity layer are in the range of 0.3 to $0.8 \mathrm{~m} / \mathrm{s}$.

\section{Numerical model and results}

Equations (5), (7), (8), (9) and (10) define a set of five equations with 5 unknown variables, being: $\mathrm{h}_{2}, \mathrm{u}_{2}, \mathrm{c}_{2}, \mathrm{q}$ and $\mathrm{q}_{\mathrm{s}}$, which can be solved for given values at the upstream boundary $(\mathrm{x}=0)$. It is most easy to specify $\mathrm{h}_{2,0}, \mathrm{c}_{2,0}$ and $\mathrm{Fr}_{2,0}$ at $\mathrm{x}=0 ; \mathrm{u}_{2,0}$ can be computed from $\operatorname{Fr}_{2,0}$ as: $\mathrm{u}_{2,0}=\mathrm{Fr}_{2,0}\left(\left(\Delta \rho / \rho_{2}\right) \mathrm{g} \mathrm{h}_{2,0}\right)^{0.5}$. Generally, it is allowed to assume $\cos \beta=1$.

The numerical model (TC-SAND; Excel application) has been used to simulate the turbidity current observed in the Zaire submarine canyon at $4000 \mathrm{~m}$ water depth (Khripounoff, 2003). The Zaire canyon (depth of about $150 \mathrm{~m}$, width of 1 to $2 \mathrm{~km}$ ) extends over about $760 \mathrm{~km}$ from the river mouth to the ocean floor (water depth of $5100 \mathrm{~m}$ ) downslope from the Congo-Angola continental shelf.

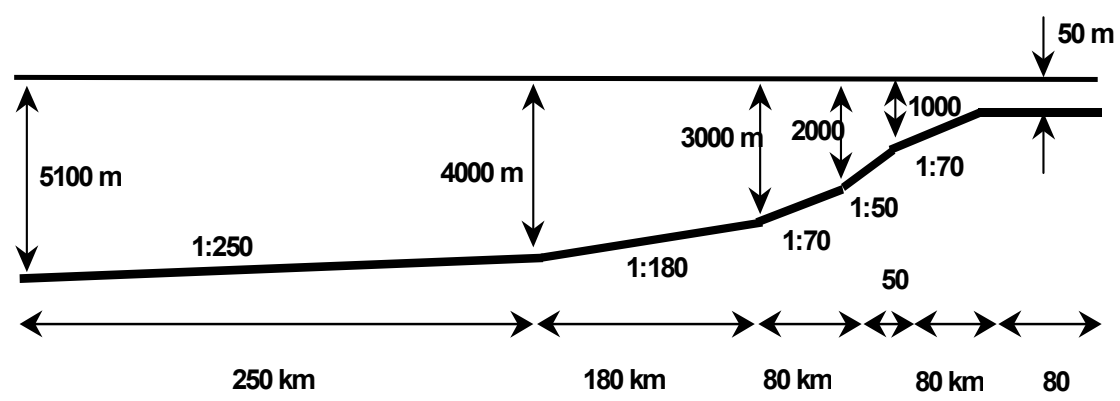

Figure 2 Longitudinal Profile along the Zaire canyon (coast on right side)

Figure 2 shows a profile along the length of the canyon. The longitudinal profile was represented by about 7500 grid points with increasing step size from $0.1 \mathrm{~m}$ 
at the entrance of the canyon to $250 \mathrm{~m}$ at the most offshore station at $640 \mathrm{~km}$. A self-accelerating turbidity current of fine sand $(0.15 \mathrm{~mm})$ could only be established by assuming the presence of a relatively steep profile (slope angle of $10^{\circ}$; slope of 1 to 6 ) over the first $200 \mathrm{~m}$. The boundary conditions are: $\mathrm{h}_{20}=0.05 \mathrm{~m}, \mathrm{c}_{20}=200 \mathrm{~kg} / \mathrm{m}^{3}, \mathrm{Fr}_{20}=2, \theta_{\mathrm{cr}}=0.06, \mathrm{C}_{\mathrm{d}}=0.004, \mathrm{C}_{\mathrm{di}}=0.0013, \alpha_{\mathrm{d}}=1$ (no damping of turbulence at upper interface), $\rho_{\mathrm{w}}=1020 \mathrm{~kg} / \mathrm{m}^{3}$, erosion coefficient $=0.0025$ (standard value). The computed results are given in Table 2 for two particle sizes $\left(\mathrm{d}_{50}=0.1 \mathrm{~mm}, \mathrm{w}_{\mathrm{s}}=0.008 \mathrm{~m} / \mathrm{s}\right.$ and $\mathrm{d}_{50}=0.15 \mathrm{~mm}, \mathrm{w}_{\mathrm{s}}=0.015$ $\mathrm{m} / \mathrm{s}$ ). Self-acceleration did not occur for sand of $0.2 \mathrm{~mm}$.

\begin{tabular}{|c|c|c|c|c|c|}
\hline Slope angle & $\begin{array}{l}\begin{array}{l}\text { Distance } \\
\text { from } \\
x=0\end{array} \\
(\mathrm{~km})\end{array}$ & \multicolumn{2}{|c|}{$\begin{array}{l}\text { Layer } \\
\text { thickness } \\
\mathbf{h}_{2}(\mathbf{m})\end{array}$} & \multicolumn{2}{|c|}{$\begin{array}{l}\text { Current velocity } \\
u_{2}(\mathrm{~m} / \mathrm{s})\end{array}$} \\
\hline $0-0.2 \mathrm{~km}$ & 0 & 0.05 & 0.05 & 0.45 & 0.45 \\
\hline $10^{\circ}$ & 0.2 & 1.8 & 1.7 & 1.05 & 0.8 \\
\hline \multirow[t]{2}{*}{$0.2-80 \mathrm{~km}$} & 1 & 5.3 & 5.7 & 0.7 & 0.5 \\
\hline & 10 & 19.2 & 18.5 & 2 & 1.5 \\
\hline \multirow[t]{2}{*}{$0.82^{\circ}$} & 30 & 52 & 49 & 4 & 3.3 \\
\hline & 50 & 87 & 82 & 5.6 & 4.7 \\
\hline \multirow{2}{*}{$\begin{array}{c}80-130 \mathrm{~km} \\
1.2^{\circ}\end{array}$} & 80 & 140 & 132 & 7.4 & 6.7 \\
\hline & 130 & 250 & 236 & 11.5 & 10.7 \\
\hline $\begin{array}{c}130-210 \mathrm{~km} \\
0.82^{\circ}\end{array}$ & 210 & 415 & 395 & 13.7 & 13.0 \\
\hline
\end{tabular}

\begin{tabular}{|c|c|c|c|c|c|c|}
\hline \multirow[t]{2}{*}{ Slope angle } & \multirow{2}{*}{$\begin{array}{l}\begin{array}{l}\text { Distance } \\
\text { from } \\
x=0\end{array} \\
(\mathbf{k m})\end{array}$} & \multicolumn{2}{|c|}{$\begin{array}{l}\text { Concentration } \\
c_{2}\left(\mathrm{~kg} / \mathrm{m}^{3}\right)\end{array}$} & $\begin{array}{l}\text { Sand transport } \\
\mathrm{q}_{\mathrm{s}}(\mathrm{kg} / \mathrm{s} / \mathrm{m})\end{array}$ & \multicolumn{2}{|c|}{$\begin{array}{l}\text { Froude number } \\
\mathrm{Fr}_{2}(-)\end{array}$} \\
\hline & & 0.1 & $0.15 \mathrm{~mm}$ & $0.1 \quad 0.15 \mathrm{~mm}$ & 0.1 & $0.15 \mathrm{~mm}$ \\
\hline \multirow{2}{*}{$\begin{array}{c}0-0.2 \mathrm{~km} \\
10^{\circ}\end{array}$} & 0 & 200 & 200 & 4.6 & 2 & 2 \\
\hline & 0.2 & 15 & 10 & 29 & 2.6 & 2.55 \\
\hline \multirow[t]{2}{*}{$0.2-80 \mathrm{~km}$} & 1 & 12 & 5 & 45 & 1.16 & 1.15 \\
\hline & 10 & 27 & 15 & 1040 & 1.16 & 1.15 \\
\hline \multirow[t]{2}{*}{$0.82^{\circ}$} & 30 & 40 & 30 & 8500 & 1.16 & 1.15 \\
\hline & 50 & 45 & 35 & $22000 \quad 13500$ & 1.16 & 1.15 \\
\hline \multirow{2}{*}{$\begin{array}{c}80-130 \mathrm{~km} \\
1.2^{\circ}\end{array}$} & 80 & 50 & 42 & $53000 \quad 37000$ & 1.16 & 1.15 \\
\hline & 130 & 50 & 47 & $145000 \quad 120000$ & 1.34 & 1.33 \\
\hline $\begin{array}{c}130-210 \mathrm{~km} \\
0.82^{\circ}\end{array}$ & 210 & 60 & 55 & 330000285000 & 1.16 & 1.15 \\
\hline
\end{tabular}

Table 2 Computed values for turbidity current in Zaire Submarine canyon; $d_{50}=0.1 \mathrm{~mm}$ and $0.15 \mathrm{~mm}$

Figure 3 shows the computed velocity, layer thickness and sand concentration for $\mathrm{d}_{50}=0.15 \mathrm{~mm}$. The turbidity current is supercritical up to $210 \mathrm{~km}$. At that location the layer thickness is about $400 \mathrm{~m}$ and the flow velocity is about 13 $\mathrm{m} / \mathrm{s}$, giving a specific water discharge of $5000 \mathrm{~m}^{2} / \mathrm{s}$. The sand transport rate at 
$210 \mathrm{~km}$ is about $250,000 \mathrm{~kg} / \mathrm{s} / \mathrm{m}$ or about $10 \mathrm{~m}^{3} / \mathrm{s} / \mathrm{m}$. The bed erosion velocities varies between $0.1 \mathrm{~mm} / \mathrm{s}$ for $u_{2}=0.5 \mathrm{~m} / \mathrm{s}$ to $3 \mathrm{~mm} / \mathrm{s}$ for $u_{2}=13 \mathrm{~m} / \mathrm{s}$. Beyond 210 $\mathrm{km}$ the slope angle of the bed is so small (see Figure 2) that the flow becomes
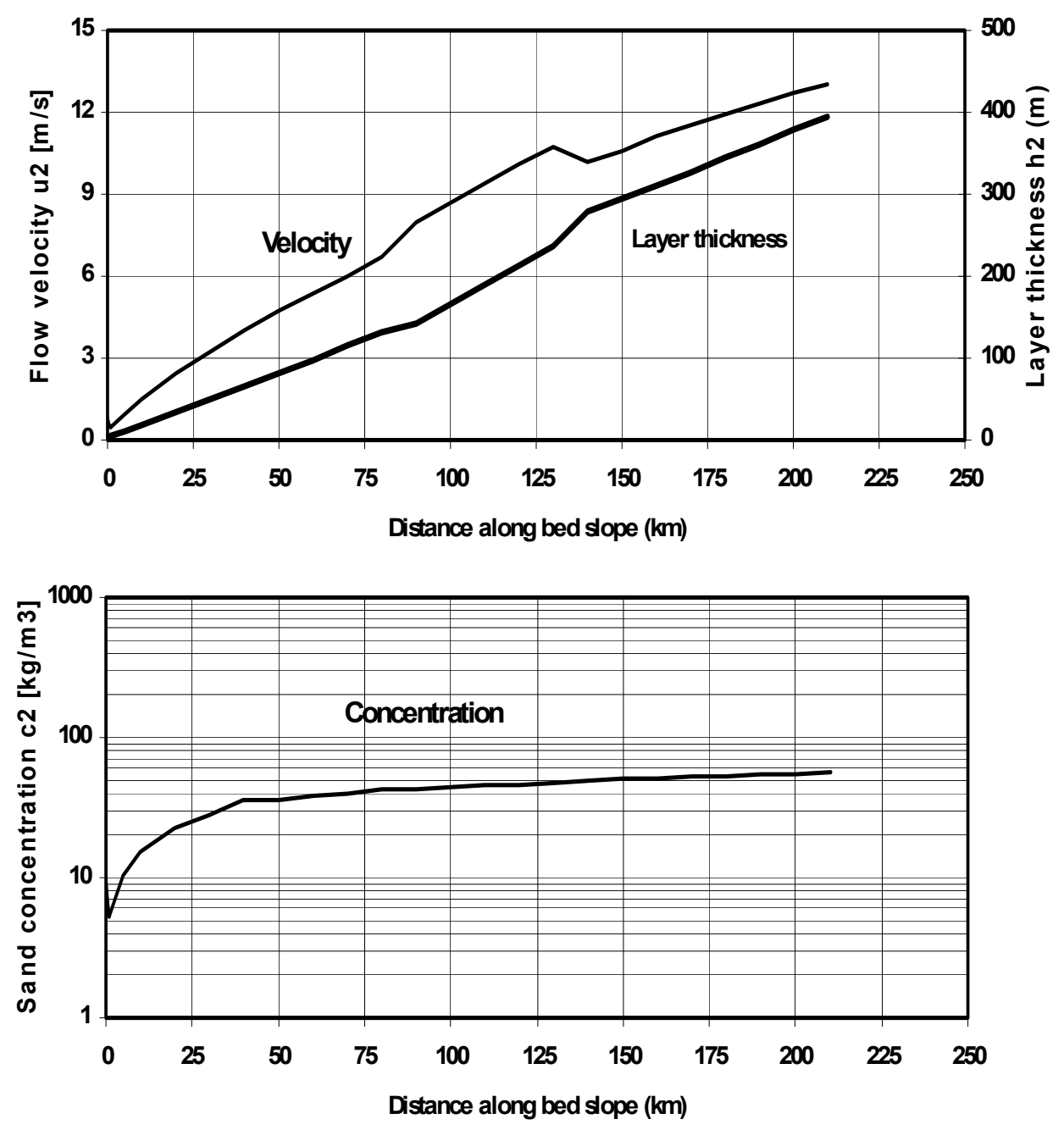

subcritical. The transition to subcritical flow may be established by an internal hydraulic jump. 
Figure 3 Computed velocity, layer thickness and sand concentration of the turbidity current along the Zaire Submarine canyon based on TCSAND model $\left(d_{50}=0.15 \mathrm{~mm}\right)$

The layer thickness in subcritical flow will be much larger (about 1000 to 2000 $\mathrm{m}$ ) and the flow velocity will be smaller (about 2 to $5 \mathrm{~m} / \mathrm{s}$ ), assuming that the specific water discharge remains about $5000 \mathrm{~m}^{2} / \mathrm{s}$ over some distance. However, the flow will gradually decay due to deposition of the suspended sediments seaward of $210 \mathrm{~km}$ (see Figure 2). The observed velocity at $360 \mathrm{~km}$ was about $1.2 \mathrm{~m} / \mathrm{s}$ at a height of 110 to $150 \mathrm{~m}$ above the bed of the canyon (in the initial phase of the turbidity current event).

In nature the layer thickness is limited by the maximum canyon depth of about $150 \mathrm{~m}$. Above this level the turbidity flow can also spread out in lateral direction resulting in a reduction of the vertical growth of the turbidity layer. These effects have been neglected in the TC-SAND model.

\section{Conclusions}

The results show that turbidity currents in coastal waters can be simulated reasonably well using a relatively simple numerical model based on a two-layer schematization. Modelling of a turbidity current generated in the Zaire Canyon (Africa) shows that the turbidity current is supercritical over about $200 \mathrm{~km}$. The behaviour of a turbidity current is dominated by the erosion of sediment from the bed into the flow. The friction coefficient of the bed also has a substantial effect because of its effect on the bed-shear stress and hence on the erosion of sediment. An increase of the erosion leads to larger concentrations in the lower layer enhancing the self-acceleration effect.

\section{References}

Akiyama, J. and Stefan, H., 1985. Turbidity current with erosion and deposition, p. 1473-1496. Journal of Hydraulic Engineering, Vol.111, No. 12

Bagnold, R.A., 1962. Auto-suspension of transported sediment; turbidity currents, p. 315-319. Proc. Royal Society, A, No. 1322, London, UK

Khripounoff, A. et al., 2003. Direct observation of intense turbidity current activity in the Zaire submarine valley at 4 ooo $m$ water depth, $p$ 151-158. Marine Geology, 194 
Mastbergen, D.R. and Van den Berg, J.H., 2003. Breaching in fine sands and the generation of sustained turbidity currents in submarine canyons. Sedimentology, Vol. 50, 625-637

Ning Chien, 1999. Mechanics of sediment transport. ASCE Press, Reston, Virginia, USA

Parker, G., Fukushima, Y., and Pantin, H.M., 1986. Self-accelerating turbidity currents, $p$ 145-181. Journal of Fluid Mechanics, Vol. 171

Sloff, C.J., 1994. Modelling turbidity currents in reservoirs. Report No. 94-5, Civil Engineering Department, Delft University of Technology, Delft, The Netherlands

Sloff, C.J., 1997. Sedimentation in reservoirs. Doc. Thesis, Civil Engineering Department, Delft University of Technology, Delft, The Netherlands

Van Rijn, L.C., 1983. Entrainment of fine sediment particles: sediment pick-up functions. Report M1531 Part IV, Delft Hydraulics, Delft, The Netherlands

Van Rijn, L.C., 1984. Sediment pick-up functions. Journal of Hydraulic Engineering, Vol. 110, No. 10; Delft Hydraulics Communication No. 370

Van Rijn, L.C., 1986. Applications of Sediment pick-up functions. Journal of Hydraulic Engineering, Vol. 112, No. 9; Delft Hydraulics Communication No. 370

Van Rijn, L.C., 1990. Principles of fluid flow and surface waves in rivers, estuaries, seas and oceans. Aqua Publications, Amsterdam, The Netherlands (WWW.AQUAPUBLICATIONS.NL)

Van Rijn, L.C., 1993. Principles of sediment transport in rivers, estuaries and coastal seas. Aqua Publications, Amsterdam, The Netherlands (WWW.AQUAPUBLICATIONS.NL)

Van Rijn, L.C., 2005. Principles of sedimentation and erosion engineering in rivers, estuaries and coastal seas. Aqua Publications, Amsterdam, The Netherlands (WWW.AQUAPUBLICATIONS.NL)

Winterwerp, J.C., Bakker, W.T., Mastbergen, D.R. and Van Rossum, H., 1992. Hyperconcentrated sand-water mixture flows over erodible bed. Journal of Hydraulic Engineering, Vol. 118, No. 11 
KEYWORDS - ICCE 2004

Title: Extreme transport of sediment due to turbidity currents in coastal waters Author: L.C. van Rijn

Abstract number: 17

Sediment transport; turbidity currents; self-acceleration 